\title{
Voltage-probe-controlled breakdown of the quantum Hall effect
}

\author{
L. W. Molenkamp, M. J. P. Brugmans, ${ }^{*}$ H. van Houten, and C. W. J. Beenakker \\ No 19502 Philips Research Laboratories, 5600 JA Eindhoven, The Netherlands \\ C. T. Foxon \\ Philips Research Laboratories, Redhill, Surrey, RH1 5HA, England
}

(Received 21 February 1991)

\begin{abstract}
We have observed that the breakdown of the quantum Hall effect in a narrow channel at high current densities can be controlled by adjusting the transmission probabilities of voltage probes at the high electrochemical-potential edge of the channel, even though contacts outside the channel are used for the measurement. We find that breakdown occurs predominantly by backscattering within the uppermost occupied Landau level.
\end{abstract}

Our understanding of the quantum Hall effect has gained considerably as a result of the use of quantum point contacts ${ }^{1}$ in the study of this phenomenon. The point contacts can selectively populate and detect the quasi-one-dimensional edge channels involved in the electron transport ${ }^{2}$ in a two-dimensional electron gas (2DEG) at high magnetic fields. The first experiments of this type involved what is now called the anomalous integer quantum Hall effect, in which the absence of scattering between the edge channels on the same edge was demonstrated on both microscopic ${ }^{3}$ and macroscopic ${ }^{4,5}$ length scales. In addition, similar experiments on the longitudinal resistance ${ }^{6}$ have provided evidence that backscattering, manifested by Shubnikov-de Haas oscillations, occurs predominantly within the highest occupied Landau level. These, and other, ${ }^{1}$ phenomena observed in the linear-response regime of vanishingly small current can be well understood on the basis of Büttiker's model of the quantum Hall effect, ${ }^{2}$ which expresses the longitudinal and Hall conductance in terms of transmission probabilities for edge channels at the Fermi level.

The breakdown of the quantum Hall effect at high current densities (the regime of nonlinear response) is considerably less well understood. Experimentally, ${ }^{7,8}$ the breakdown is usually studied in a narrow $(\sim 1 \mu \mathrm{m})$ channel or constriction. In such structures large Hall fields can be generated at moderate current levels $(\sim 0.1-1$ $\mu \mathrm{A}$ ). Several mechanisms have been proposed (cf. Ref. 9 and references therein), but the interpretation of the experiments is not unambiguous. We report results of an experimental study of the breakdown of the quantum Hall effect in a novel geometry, i.e., a narrow channel fitted with adjustable point contact voltage probes. We use the voltage probes to adjust the equilibration of the highest occupied Landau level with the lower levels. ${ }^{10}$ Our data provide evidence that breakdown occurs predominantly through selective backscattering of electrons in the highest Landau level.
Figure 1 gives a layout of the structure used in this work. The sample is fabricated from a high mobility (Al,Ga)As heterojunction wafer containing a 2DEG with a sheet electron concentration $n_{s}=3.47 \times 10^{11} \mathrm{~cm}^{-2}$ and a mobility $\mu=1.4 \times 10^{6} \mathrm{~cm}^{2} / \mathrm{Vs}$. In the figure, crosses indicate Ohmic contacts to the 2DEG; the hatched areas are split gates that are used to electrostatically define a channel of width $W_{\mathrm{ch}}=4 \mu \mathrm{m}$ and length $L_{\mathrm{ch}}=18 \mu \mathrm{m}$. Two opposite pairs of quantum point contacts are defined on the top $\left(t_{1}\right.$ and $\left.t_{2}\right)$ and bottom $\left(b_{1}\right.$ and $\left.b_{2}\right)$ edge of the channel, with a separation of $3 \mu \mathrm{m}$ between adjacent point contacts. The gate voltages are adjusted such that adjacent point contacts have equal resistance $\left(R_{t_{1}}=R_{t_{2}} \equiv R_{t}\right.$ and $\left.R_{b_{1}}=R_{b_{2}} \equiv R_{b}\right)$. We present results obtained for a sample temperature of $1.65 \mathrm{~K}$, and a fixed magnetic field $B=3.45 \mathrm{~T}$, corresponding to a filling factor $\nu=n_{s} h / 2 e B=2.0$ in the narrow channel. (Because of electrostatic depletion, $n_{s}$ in the channel is somewhat smaller than in the bulk 2DEG, where $\nu=2.08$ at $3.45 \mathrm{~T}$.) A current $I$ is passed through the channel from Ohmic contact 1 to 6 . With the magnetic field direction as indicated in the figure, and for positive

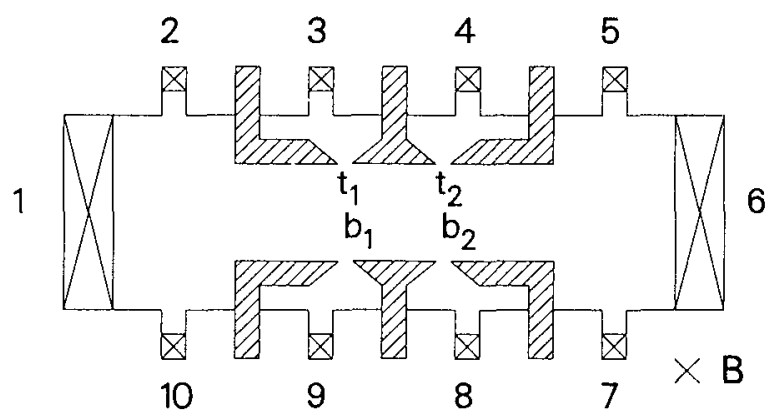

FIG. 1. Layout of the Hall bar (not to scale), containing a narrow channel (of width $4 \mu \mathrm{m}$ and length $18 \mu \mathrm{m}$ ) with point contact voltage probes ( $3 \mu \mathrm{m}$ apart). Positive current flows from Ohmic contact 1 to 6 . 
currents, the top edge of the channel has the highest electrochemical potential for electrons ( 1 e , it is chaiged negatively) The differential resistance between Ohmic con tacts $\imath$ and $\jmath, R_{\imath \jmath}=d V_{\imath \jmath} / d I$, with $V_{\imath \jmath} \equiv V_{\imath}-V_{\jmath}$, is measured using a low-frequency lock-in technique Differential resistance data have been obtained for four different sets of values of the point contact resistances $R_{t}$ and $R_{b}$ These sets colrespond to different numbers $\left(N_{t}, N_{b}\right)$ of spin-degenerate edge channels that are fully transmitted through the point contacts on either side of the channel (note that $R_{t, b}=h / 2 e^{2} N_{t, b}$ ) The configurations used are $\left(N_{t}, N_{b}\right)=(2,2),(1,2),(2,1)$, and $(1,1)$

In Fig 2 we show the current dependence of the longitudinal differential resistance of the channel $\left(R_{25}\right)$, measured with voltage probes adjacent to the channel Contacts 2 and 5 are ideal voltage probes in that they equally populate all avalable edge channels ${ }^{2}$ The data in this figure reveal a pronounced influence of the adjustment of the point contacts at the channel boundaries on the observed Hall breakdown characteristics For the set $\left(N_{t}, N_{b}\right)=(2,2)$ (solid curve) the breakdown occuis at a relatively small curıent, whereas for $\left(N_{t}, N_{b}\right)=(1,1)$ (dash-dotted curve) considerably larger currents are required to obtain breakdown In both cases, $R_{25}$ is symmetric in $I$, at least at relatively small current levels In contrast, the breakdown curves for the mixed sets $\left(N_{t}, N_{b}\right)=(1,2)$ (dashed curve) and $(2,1)$ (dotted curve) are asymmetılc Moreover, these latter culves comcide with parts of those obtained for equal point contacts $[(2,2)$ and $(1,1)]$ in a specific manner This coincidence occurs whenever the point contacts at the high-potentıal edge have been adjusted similarly (a condition which depends on the current direction) Only the adjustment of the voltage probes on the high-potential edge influences the breakdown characteristics Since the onset of break-

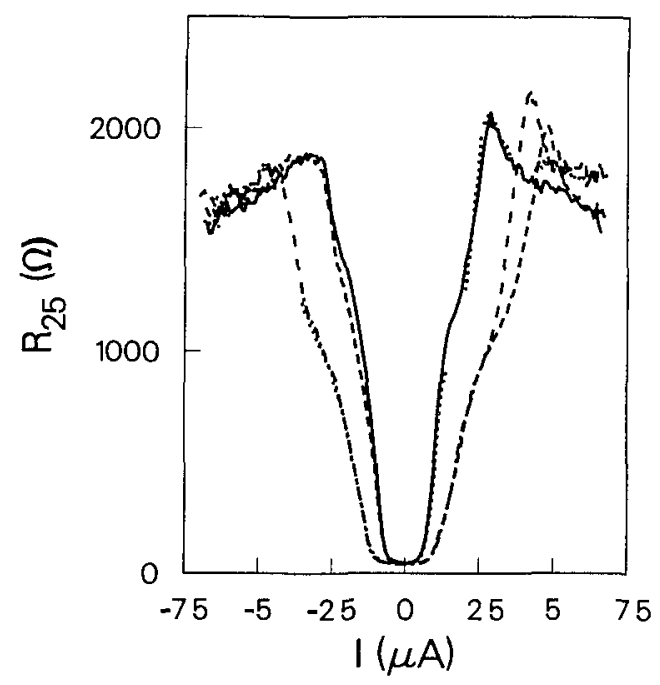

FIG 2 Longitudinal differential resistance $R_{25}$ vs current $I$ for four different configurations of the point contact voltage probes $\left(N_{t}, N_{b}\right)=(2,2)$ (solid curve), $(1,2)$ (dashed curve), $(2,1)$ (dotted curve), and $(1,1)$ (dash-dotted curve) down occurs at smaller currents when the probes transmit both edge channels, we can conclude that the breakdown is enhanced by probe-induced equilibration between the higher and the lower edge channels, but only when this equilibration occurs at the high-potential edge

More detalled information on the influence of the point contact resistance on the breakdown characteristics can be obtained from Fig 3, where we show the dependence of $R_{25}$ on the gate voltage $V_{\text {gate, } t}$ used to define point contacts $t_{1}$ and $t_{2}$, for various values of the current, and for $N_{b}=1$ For comparison, the upper panel shows the gate-voltage dependence of the two-termmal resistance $R_{t}$ of point contacts $t_{1}$ and $t_{2}$ For negative currents (thin lines in the breakdown curves), the bottom edge is at the highest electrochemical potentral The data in Fig 3 show clearly that in this case the breakdown signal does not depend on the top gate voltage $V_{\text {gate } t}$ (except for $V_{\text {gate } t}$ between 0 and $-05 \mathrm{~V}$, where the channel is not well defined) For positive currents, the top edge is at the highest potential, and $R_{25}$ does depend strongly on $V_{\text {gate, } t}$ The breakdown is enhanced when $N_{t}=2$ (colresponding to $R_{t} \sim 6 \mathrm{k} \Omega$ ) For more negatıve gate voltages $\left(N_{t}<2\right)$ the bieakdown resistance $R_{25}$ decreases, eventually reaching the value found for negative currents These data confirm our conclusions that only the adjustment of the point contacts on the high-potential edge is of
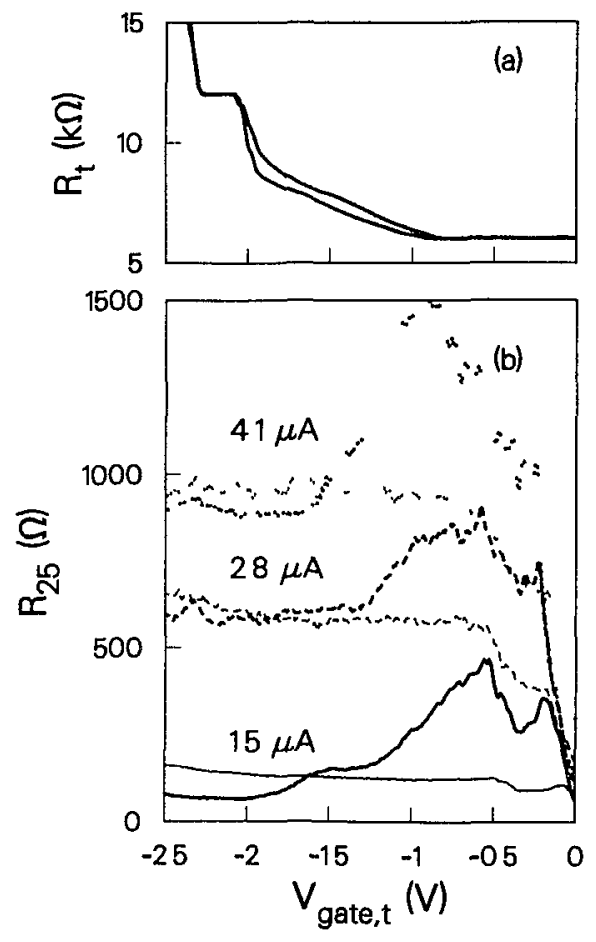

FIG 3 (a) Resistance $R_{t}$ of point contacts $t_{1}$ and $t_{2}$ as a function of gate voltage (b) Dependence of the longitudinal differential resistance $R_{25}$ on the top gate voltage $V_{\text {gate }} t$ used to define point contacts $t_{1}$ and $t_{2}$ Data are shown for three current levels, and for both current directions, thick curves refer to positive, thinner curves to negative currents In all cases $N_{b}=1$ 
importance, and that enhanced breakdown occurs when both edge channels are transmitted through these point contacts.

Figure 4 summarizes our experiments on the threeterminal differential resistance $R_{13} \equiv d V_{13} / d I$, measured using a quantum point contact at the top edge of the channel (3) as one voltage probe, and one of the current contacts (1) as the other. The data shown in this figure exhibit (a)symmetries similar to those in Fig. 2 However, in contrast to Fig. 2, in this case we do observe a strong effect on the breakdown data of the adjustment of the point contact at the top edge (1.e, of $N_{t}$ ) when it is at the lowest electrochemical potential (negative $I$ ): when the highest occupied edge channel is not transmitted $\left(N_{t}=1\right), R_{13}$ is, at small negative $I$, much larger than for $N_{t}=2$ This effect arses because contact 3 is not an ideal voltage probe, its potentral being determined by the transmission of point contact $t_{1}$ (cf. the experiments on the anomalous integer quantum Hall effect ${ }^{3-5}$ ). Our data imply that, under breakdown conditions, the edge channels with quantum number $n \geq 2$ at the low-potential edge are not in equilibrium with the lowest $(n=1)$ edge channel. ${ }^{11}$ This is direct evidence of selectrve backscattering in the highest occupied Landau levels, reminiscent of the selectivity causing ${ }^{6,12}$ the Shubnikov-de Haas oscillations in the linear regime. On further increasing the negative current in the channel, the anomalously large value of $R_{13}$ suddenly drops to the value measured for $N_{t_{1}}=2$. Since the Hall voltage is too small to substantially affect the resistance of point contact $t_{1}$, our observation implies that for these current levels the edge channels at the low-potential edge equilibrate on distances short compared to the channel length. A breakdown of adiabatic transport at large current densities was also reported by Komiyama et al., ${ }^{4}$ and was attributed to the large difference in electrochemical potential between two adjacent edge channels. ${ }^{13}$

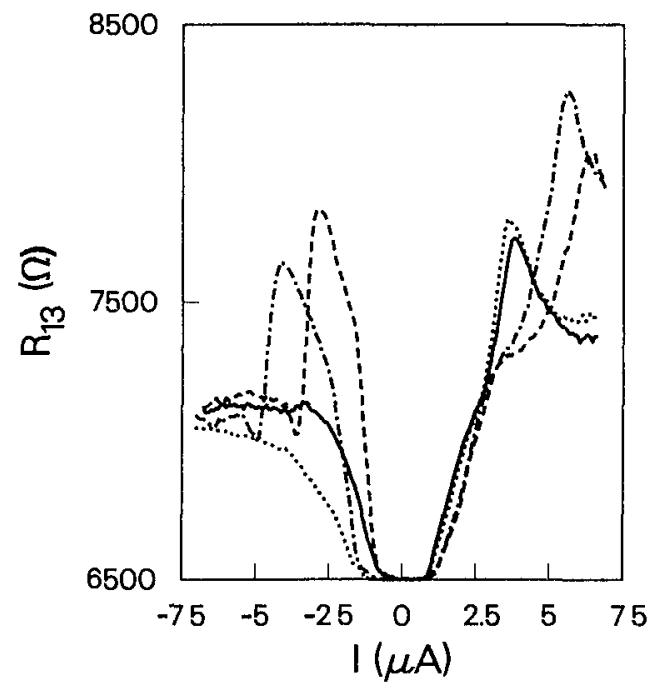

FIG. 4. Three-terminal differential resistance $R_{13}$ vs current $I$ for the same point contact configurations as in Fig 2 (with the same coding of the curves).
Hall-voltage induced selective backscattering can be qualitatively understood, as follows. Figure 5(a) depicts the variation of the energy of the highest Landau level along a cross section of the narrow channel at finite positive current. ${ }^{9}$ The thick lines symbolize the occupied edge states: the solid line indicates electrons mov-

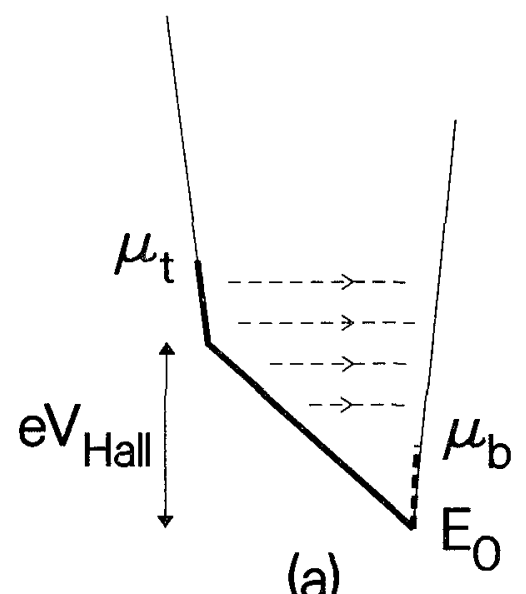

(a)
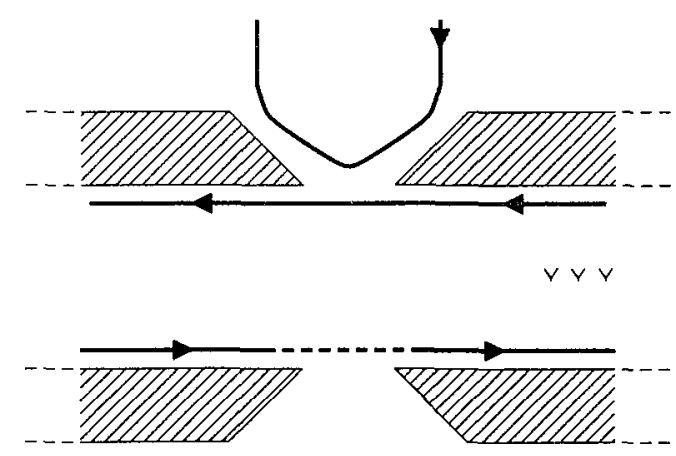

(b)

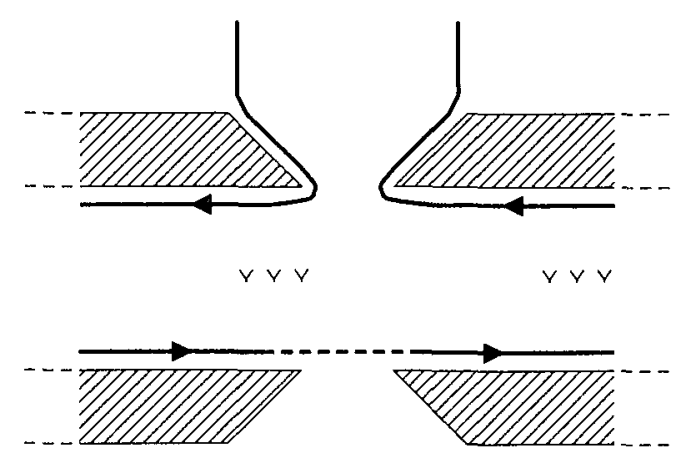

(c)

FIG. 5. (a) Schematic energy diagram of the highest occupied Landau level along a cross section of the narrow channel (b) and (c) Edge channels in the highest occupied Landau level. When $N_{t}=1$, as in (b), backscattering occurs predominantly at the entrance of the channel, while for $N_{t}=2$, as in (c), equilibration between the Landau levels in the region behind the point contact on the high-potential edge causes a second opportunty for backscattering. The transmission of the edge channel by the point contact on the potential edge (dashed) is irlelevant. 
ing along the high-potential edge, and the dashed line refers to electrons moving along the low-potential edge in the opposite direction. The arrows indicate backscattering from the high-potential $(t)$ to the low-potential (b) edge. For states at the high-potential edge with an energy $E$ such that $\mu_{b}<E \leq E_{0}+e V_{\text {Hall }}$, where $E_{0}$ is the bottom of the Landau level, we have a situation where the overlap of the wave function of these occupied states and empty edge states on the low-potential edge can be continuously increased by increasing the current in the channel, and consequently the Hall voltage $V_{\text {Hall }}$. This results in enhanced backscattering. Irrespective of the details of the backscattering mechanism (direct versus impurity assisted, inter- or intra-Landau level), one can state that for lower Landau levels the current, in the same Hall voltage regime, has a much smaller influence on the overlap between occupied high-potential and empty low-potential edge states. Consequently, Hall-voltage induced backscattering occurs predominantly in the highest occupied Landau level (and, possibly, into still higher unoccupied Landau levels ${ }^{11}$ ).

The observed effects of the adjustment of the point contacts on the breakdown can be explained by means of Figs. 5(b) and 5(c), which show the location of the edge channel wave function in the higher Landau level for $N_{t}=1$ and 2, respectively (the lower Landau level has been omitted for clarity). In both situations, electrons entering the narrow channel region along the top edge in the highest Landau level are backscattered due to the proximity of the edge channel at the opposite edge. The reverse process is also possible, so that eventually a steady-state situation is reached, with a partially depleted population of the higher edge channel at the high- potential edge (corresponding to a certain nonzero longitudinal resistance). This steady state is reached close to the channel entrance. If, however, the highest Landau level is transmitted by the point contact [Fig. 5(c), $N_{t}=2$ ], the edge channels are equilibrated. This causes a repopulation of the partially depleted higher channel, and consequently a second opportunity for backscattering, which did not exist for $N_{t}=1$.

The observed asymmetry in the breakdown curves between $\left(N_{t}, N_{b}\right)=(2,1)$ and $(1,2)$ is intrinsically a nonlinear response effect, because it implies a dependence of the resistance on the direction of the current. We have attempted to model our observations by incorporating an energy-dependent backscattering probability in the standard Landauer-Büttiker formalism. ${ }^{2}$ While we do find asymmetries in the breakdown curves depending on the direction of the current, our over-simplified model does not yield a satisfactory quantitative agreement with the experimental curves.

In conclusion, our experimental results demonstrate that breakdown of the quantum Hall effect in a narrow channel proceeds predominantly via selective backscattering within the highest Landau levels.

The authors would like to thank B. W. Alphenaar, P. C. van Son, and A. A. M. Staring for useful discussions, and C. E. Timmering and M. A. A. Mabesoone for expert technical assistance. We acknowledge the stimulating support of M. F. H. Schuurmans. This research was partly funded under the European Strategic Programme for Research and Development in Information Technology basic research action Project No. 3133.
*Also at Eindhoven University of Technology, $5600 \mathrm{MB}$ Eindhoven, The Netherlands.

${ }^{1}$ For a review, see H. van Houten, C. W. J. Beenakker, and B. J. van Wees, in Semiconductors and Semimetals, edited by M. Reed (Academic, New York, in press).

${ }^{2}$ M. Büttiker, Phys. Rev. B 38, 9375 (1988).

${ }^{3}$ B. J. van Wees, E. M. M. Willems, C. J. P. M. Harmans, C. W. J. Beenakker, H. van Houten, J. G. Williamson, C. T. Foxon, and J. J. Harris, Plyys. Rev. Lett. 62, 1181 (1989).

${ }^{4}$ S. Komiyama, H. Hirai, S. Sasa, and S. Hiyamizu, Phys. Rev. B 40, 12566 (1989).

${ }^{5}$ B. W. Alphenaar, P. L. McEuen, R. G. Wheeler, and R. N. Sacks, Phys. Rev. Lett. 64, 677 (1990).

${ }^{6}$ B. J. van Wees, E. M. M. Willems, L. P. Kouwenhoven, C. J. P. M. Harmans, J. G. Williamson, C. T. Foxon, and J.

J. Harris, Phys. Rev. B 39, 8066 (1989).

${ }^{7}$ J. R. Kirtley, Z. Schlesinger, T. N. Theis, F. P. Milliken, S. L. Wright, and L. F. Palmateer, Phys. Rev. B 34, 5414 (1986).

${ }^{8}$ P. G. N. de Vegvar, A. M. Chang, G. Timp, P. M. Mankiewich, J. E. Cunningham, R. Behringer, and R. E. Howard, Phys. Rev. B 36, 9366 (1987).
${ }^{9}$ P. C. van Son, G. H. Kruithof, and T. M. Klapwijk, Phys. Rev. B 42, 11267 (1990).

${ }^{10}$ The use of point contacts to equilibrate edge channels was demonstrated in the linear transport regime by B. J. van Wees, L. P. Kouwenhoven, E. M. M. Willems, C. J. P. M. Harmans, J. E. Mooij, H. van Houten, C. W. J. Beenakker, J. G. Williamson, and C. T. Foxon, Phys. Rev. B (to be published).

${ }^{11}$ We write $n \geq 2$ instead of $n=2$, because inter-Landaulevel scattering becomes energetically allowed at large Hall voltages. Our experimental data allow the possibility of a nonequilibrium population involving also Landau levels with $n>2$ (which are not populated in equilibrium).

${ }^{12}$ P. L. McEuen, A. Szafer, C. A. Richter, B. W. Alphenaar, J. K. Jain, A. D. Stone, and R. G. Wheeler, Phys. Rev. Lett. 64, 2062 (1990).

${ }^{13}$ S. Komiyama, H. Hirai, M. Ohsawa, H. Matsuda, S. Sasa, and T. Fujii, in Proceedings of the 20th International Conference on the Physics of Semiconductors, edited by E. M. Anastassakis and J. D. Joannopoulos (World Scientific, Singapore, 1990), p. 1150. 